\title{
Integrative Medicine and Drug-Induced Vestibulopathy following Brucellosis Treatment: Clinical Practice
}

\author{
Majid Avijgana Mahtab Avijgand Mohamad Reza Najafib \\ Najmeh Salek $^{a} \quad$ Azin Momeni $^{a} \quad$ Shiva Shabani ${ }^{c} \quad$ Fahimeh Salehi $^{a}$ \\ Departments of a Traditional Persian-Iranian Medicine and ${ }^{\mathrm{b}}$ Neurology, and ${ }^{\mathrm{C}}$ Infectious \\ Diseases, Isfahan University of Medical Sciences, Isfahan, Iran; dUniversity of Newcastle, \\ Newcastle, N.S.W., Australia
}

\section{Key Words}

Acupuncture · Brucellosis · Drug complication · Fever of unknown origin · Integrative medicine $\cdot$ Streptomycin $\cdot$ Traditional medicine $\cdot$ Vestibulopathy

\section{Abstract}

Brucellosis is still one of the most common causes of fever of unknown origin (FUO) in endemic areas and is associated with several complications. Here, we report a patient with a diagnosis of brucellosis and FUO who developed a rare complication of vestibulopathy in the course of treatment and follow-up. We treated the vestibulopathy with acupuncture, and during 10 days of treatment, the irreversible complication of vestibulopathy disappeared. To the best of our knowledge, this is the first case report of brucellosis with vestibulopathy treated with traditional medicine that achieved complete improvement within 10 days.

\section{Introduction}

Brucellosis is a zoonotic disease with an incidence rate of more than half a million per year worldwide that causes systemic symptoms and can involve many organs and tissues. The major source of infection is consumption of unpasteurized dairy products and occupational contacts [1]. Brucellosis is widespread in the countries of the Mediterranean region, the Persian Gulf, the Indian subcontinent, parts of Mexico, and Central and South America [2]. Despite the disease control in many countries, it remains endemic in Iran, especially in the 
Avijgan et al.: Integrative Medicine and Drug-Induced Vestibulopathy following Brucellosis Treatment: Clinical Practice

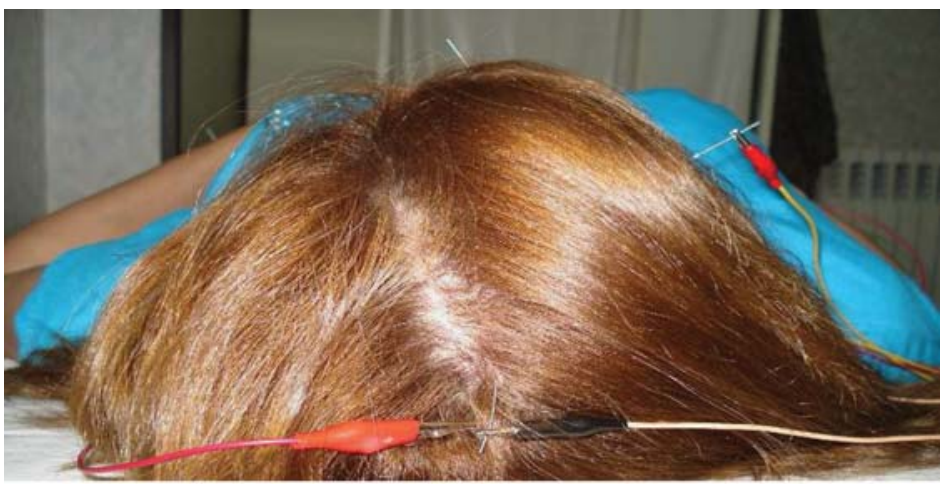

Fig. 1. Place and method of double puncture of DU20.

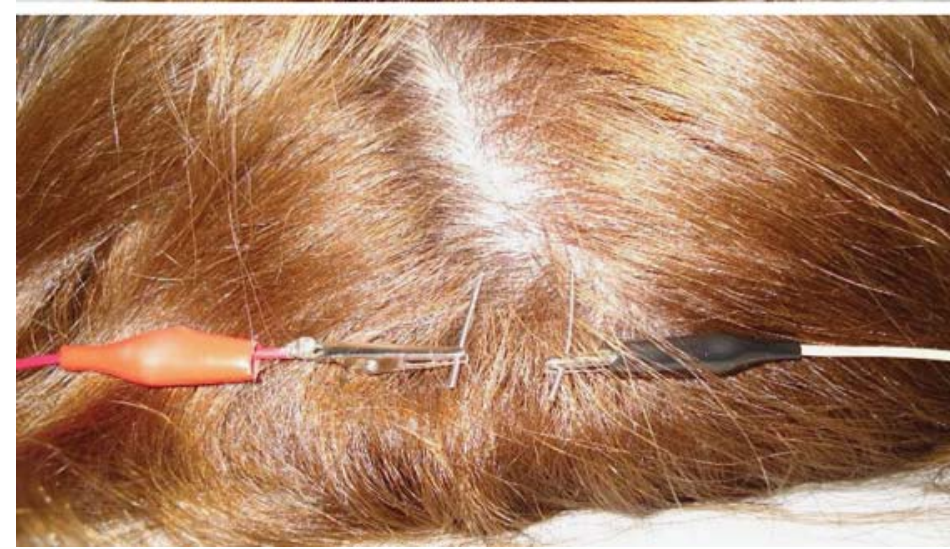

Isfahan province, where Brucella melitensis has a high prevalence rate in humans and animals [3].

Brucellosis may commonly be misinterpreted because of its extremely variable clinical picture, and in some chronic cases, negative microbiological and serological results [4]. For these reasons, the clinical experience and suspicion of infection may sometimes be the only method of diagnosis, especially in endemic regions.

This report presents a patient suffering from chronic brucellosis, manifested as a fever of unknown origin (FUO) and spondylitis of the L3-L4 vertebrae with negative blood and tissue cultures, negative agglutinating titers and nonspecific radiological signs of 2 months' duration without a specific diagnosis. This case provides valuable additional data. (1) Although several other infectious diseases appear as a cause of FUO, brucellosis in particular should be considered as a differential diagnosis especially in endemic areas. (2) In endemic areas, low titers of standard tube agglutination (STA) and 2-mercaptoethanol (2ME) tests should be considered as an indication of brucellosis, especially in FUO cases. (3) Should these titers be raised after a successful treatment? (4) Vestibulopathy is a rare adverse effect in comparison to hearing loss due to aminoglycosides, especially streptomycin, in which the complementary medicine such as acupuncture can be considered as a possible alternative treatment. (5) In acupuncture, one should usually connect two points, for example the DU20 and Ex-HN 5 points, with one cord of electroacupuncture, but because of the fact that the DU20 point is in the midline and the Ex-HN 5 points are in the bilateral temporal area, it was not possible to connect one point (DU20) to two points (left- and right-side Ex-HN 5). Since we should always connect one wire to the same side of the body in electroacupuncture, we inserted 2 needles as far as $2 \mathrm{~mm}$ from the DU20 point on the right and left sides and connected each one of them to the same side of the Ex-HN 5 point (fig. 1). 


\section{Integrative Medicine}

International
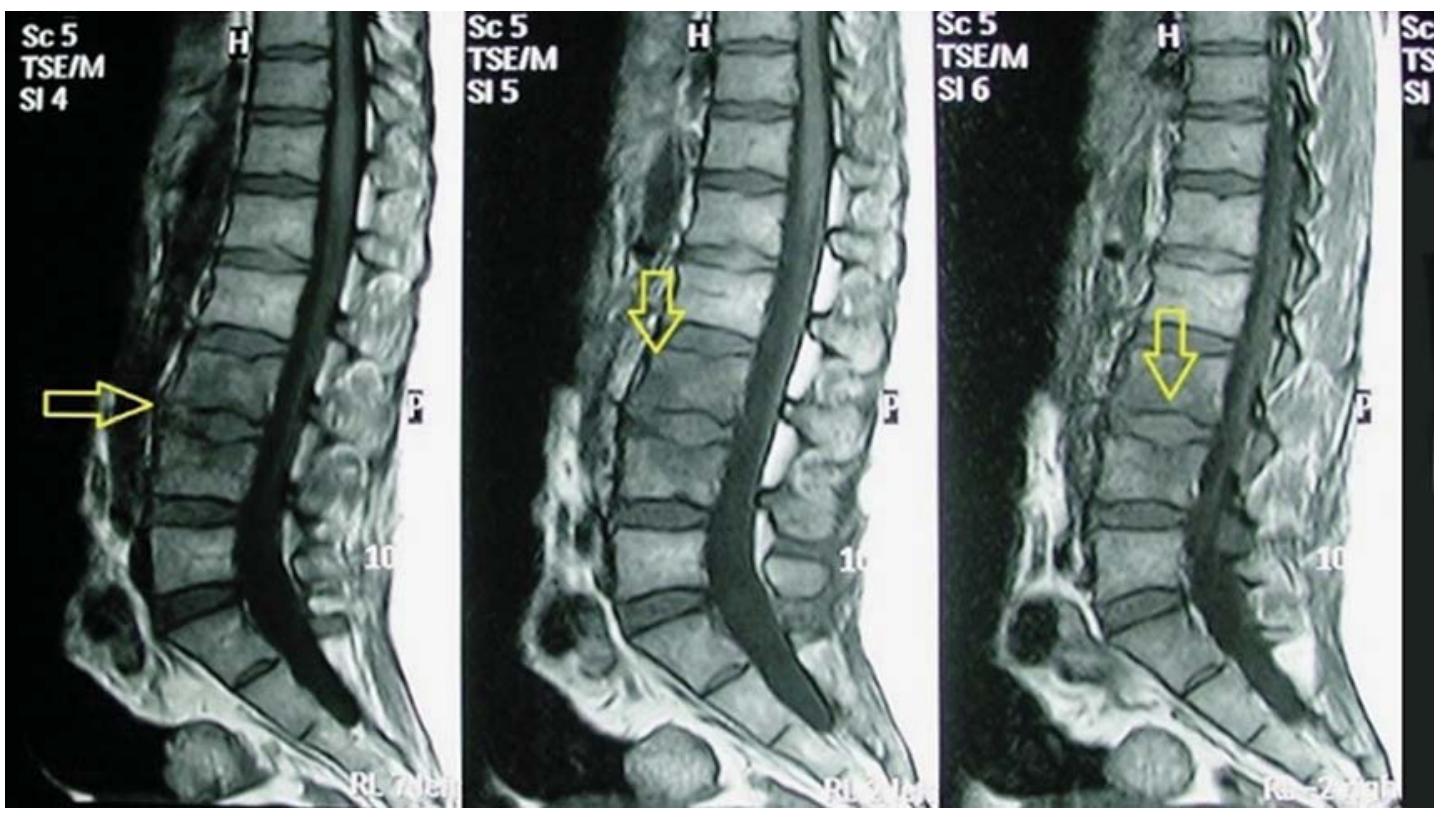

Fig. 2. Bulging of L1-L2, L3-L4 intervertebral disks and abnormal signal intensity of L3-L4 suggesting local spondylitic changes and osteomyelitis.

\section{Case Presentation}

The patient is a 47-year-old housewife suffering from chills, fever and backache. Her problems began 2 months prior to admission to the hospital with a chilly sensation and fever of $40^{\circ} \mathrm{C}$, which occurred once a day during the first week of the disease. The fever and chills in the second week occurred twice a day. In the third week, the patient started to complain about backache and visited a physician who put her on penicillin, but there was no relief in the patient's symptoms. After 1 month of suffering from these symptoms, fever and chills began occurring at a frequency of three times a day, and the severity of low back pain worsened.

In the 2 nd month of the disease, she was admitted for further investigations. Physical examination at this time was normal except for a fever of $40^{\circ} \mathrm{C}$. Results of laboratory tests made on admission were as follows: erythrocyte sedimentation rate, $85 \mathrm{~mm} / \mathrm{h}$; C-reactive protein, qualitative positive (+++); STA for brucellosis or Wright test, 1/40; the Widal test was negative; PPD skin test was negative; rheumatological tests (ANA, P ANCA, C ANCA, GBM Ab, anti-dsDNA, RF, C3, C4 and CH50) were all normal; serum protein electrophoresis, $\alpha_{1}$ increased. The other laboratory data were as follows: negative blood culture; normal echocardiography (report for diagnosis of endocarditis); normal thin peripheral blood smear for malaria; normal chest X-ray; normal mammography; normal sonography of abdomen and pelvic; normal skull X-ray; radiographs of the spine showed mild reduction in the height of the body of the L2 vertebra with a mild irregularity of the end plate. Sclerosis or lytic lesions were not seen.

The magnetic resonance imaging scan of the spine revealed a mild reduction in the height of the body of L2 with mild irregularity of the end plate, bulging of the L1-L2, L3-L4 intervertebral disks and an abnormal signal intensity of L3-L4 suggesting local spondylitic changes, and osteomyelitis (fig. 2). The patient was discharged after a week with a suggestion 
to undergo lumbar puncture and bone biopsy of the L3 vertebra and a whole body bone scan. No specific treatment was given. The lumbar puncture was normal (CSF direct smear: WBC 10-12; RBC: many, and no bacterial growth). The whole body bone scan showed an active bone lesion of L3-L4 suggesting L3-L4 osteomyelitis. The Brucella IgG ELISA was 1.1 (negative: <9) and Brucella IgM ELISA 10.7 (borderline: 9-11). A sonography-guided bone biopsy from the L3 vertebra was performed. Histopathological examination of the specimen also suggested acute and chronic osteomyelitis, and mycobacterium tuberculosis DNA PCR was negative.

Two weeks later, the patient was readmitted to our hospital to clarify the cause of her condition. Results of the laboratory tests performed on the recent admission 2 months after the start of her chilly sensation and fever were as follows: WBC count, 7,100, and 76\% neutrophils; erythrocyte sedimentation rate, $129 \mathrm{~mm} / \mathrm{h}$; C-reactive protein, +++; STA test for Brucella (Wright test), 1/80; 2ME test, 1/80; blood culture was negative again, and transesophageal echocardiography was normal. In spite of the failure to prove bacterial infection, a diagnosis of hematogenous osteomyelitis was made, and treatment with meropenem and vancomycin was started, but after 5 days, there was no change in the patient's symptoms. Unresponsiveness to the antibacterial agents led us to revise our diagnosis.

By considering the consumption of unpasteurized goat's milk 1 month before the start of her chilly sensation, fever and also low titers of STA (1/80), a probable diagnosis of brucellosis was made. In an endemic area, the diagnostic titer of STA is $1 / 160$, but because she lived in a nonendemic area and based on the national protocol [3], a titer of 1/80 was diagnosed. The patient was started on streptomycin ( $1 \mathrm{~g} /$ day), tetracycline $(2 \mathrm{~g} /$ day $)$, and rifampin $(900$ $\mathrm{mg} /$ day) with a high suspicion of brucellosis spondylitis. Simultaneously, the antibacterial agents were discontinued. After 6 days of anti-Brucella treatment, the fever subsided with gradual disappearance of back pain. After 25 days of treatment, the patient became pain free, and walking and bending had almost returned to normal.

A month after initiating treatment, the results of the laboratory tests were as follows: WBC count, 6,900, and 61\% neutrophils; Hb, 10; platelets, 282,000; ESR, 60; Wright test, $1 / 160$, and $2 \mathrm{ME}, 1 / 40$. After 2 months of follow-up, there was no fever or low back pain. Follow-up magnetic resonance imaging of the lumbar spine showed a mild reduction in the height of the body of L3 and L4. Abnormal signal intensity in the body of L3 and L4 with involvement of the L3/L4 intervertebral disk and a small anterior paravertebral soft tissue mass lesion were seen, suggesting lumbar spondyloarthritis, and there was evidence of osteomyelitis of the body of L3 and L4 with L3/L4 discitis.

Our plan was to continue the antibrucellosis medication for at least 6 months. After 1 month of initiation of treatment, she had symptoms of disequilibrium, true vertigo and falling to the left side after standing on both legs for more than $40 \mathrm{~s}$. After neurological consultation and investigations, the diagnosis was drug-induced (streptomycin) irreversible vestibulopathy. On neurological examination, action tremor of the hand, a positive Romberg test and ataxic gait were observed.

The Romberg test is a test for evaluation of the body's sense of positioning. The Romberg test assesses by asking the patient to stand erect with feet together and eyes closed. The test is positive if the patient sways or falls while the patient's eyes are closed. The Romberg test in this patient was positive before treatment. The routine tests for the ability of the body to stay in balance are the Romberg examination and single-leg upright standing [5]. A quantitative evaluation of the measurable equilibrium examination may be the duration of the patients' static-dynamic equilibrium function while standing on a single foot or two feet with eyes closed, and the walking steps [5]. The equilibrium examinations indicated that her gait was normal in slow steps, but during fast gait or running, she was prone to fall to the left side after about 3 steps (fig. 3). She fell after $42 \mathrm{~s}$ of standing on both legs with arms stretched 
Fig. 3. Left deviation after 3 steps of running (the patient is supported by her daughter).

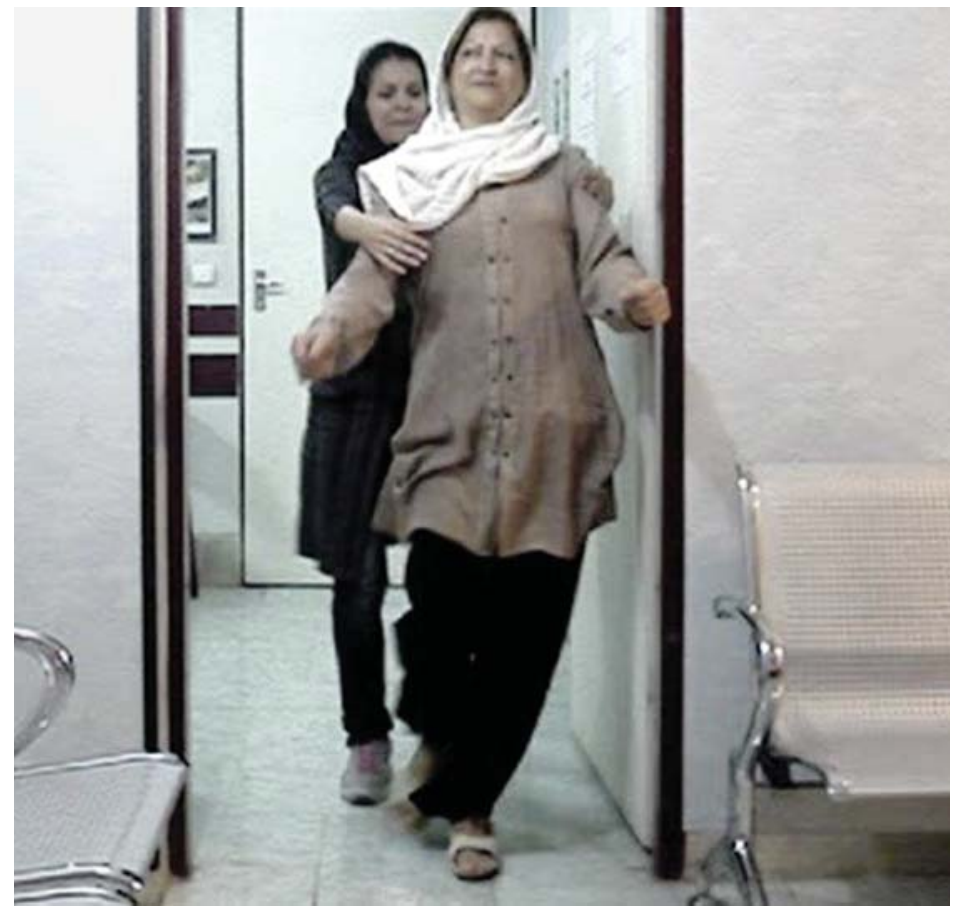

forward and open eyes, and after $12 \mathrm{~s}$ her right hand had tremor. In the same evaluation for her equilibrium with closed eyes (Romberg test), she fell after $8 \mathrm{~s}$ (fig. 4). Then, streptomycin was discontinued, and we suggested to the patient to undergo acupuncture treatment, which she accepted as the final method of treatment.

\section{Acupuncture}

\section{Diagnosis}

In this case, there was no modality for treatment, and based on the neurological consultation, she would have to wait for the outcome and a normal course of vestibulopathy, which may last several months or years. But according to traditional medicine and traditional Chinese medicine (TCM), wind (vertigo) is due to a deficiency-related phlegm obstruction of the mind and is the cause of abnormal gait and vestibulopathy.

In this case, vertigo occurs due to the vacuity of the brain, stagnation and turbid phlegm obstruction [5]. Phlegm is a viscous turbid pathological product that can accumulate in the body, causing a variety of diseases [6]. When there is a qi deficiency, there is a deficiency of qi movement [7], which is responsible for the movement of body fluid. Thus, in the case of qi deficiency, the brain is not nourished, vacuity occurs, and phlegm obstructs the brain.

On TCM history taking, the patient was assessed as an anxious healthy female with the following symptoms: SHEN (lower back pain, tidal fever, 5 heat soles, dizziness, poor memory, poor concentration, no thirst and/or hot water); FEI (chills/fever, dry skin); PI (bleeds easily, excess appetite, bloated after eating, abdominal distention, prolapsed organs, feels cold, increased urination volume); XIN (mental restlessness, ulceration/swelling of mouth or tongue), and GAN (bitter, lower abdominal pain, numbness of extremities, depression, belching). According to the TCM criteria, the patient syndrome differentiation was KI qi deficiency, SP yang deficiency and mild liver blood deficiency. 


\section{Integrative Medicine}

International

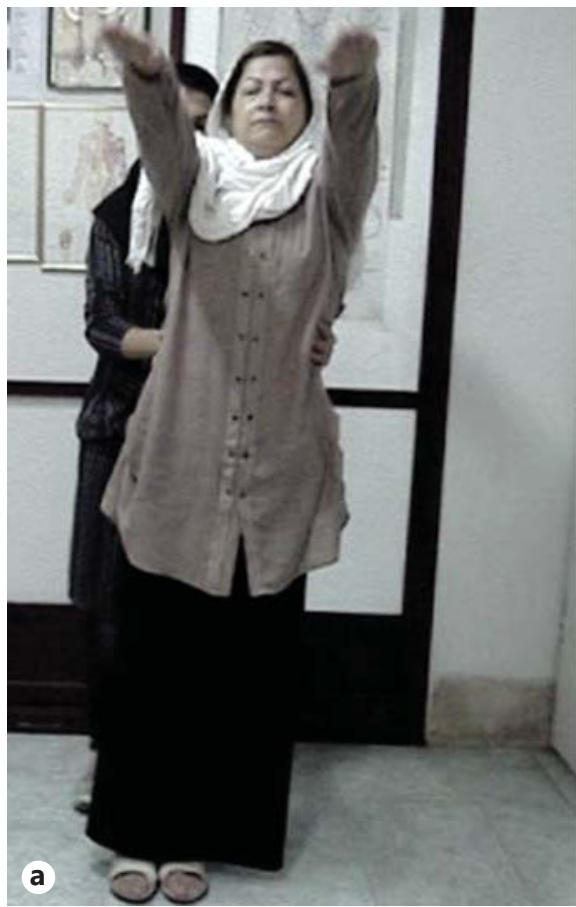

Integr Med Int 2014;1:176-184

DOI: $10.1159 / 000371800$

2015 S. Karger AG, Basel www.karger.com/imi

Avijgan et al.: Integrative Medicine and Drug-Induced Vestibulopathy following Brucellosis Treatment: Clinical Practice

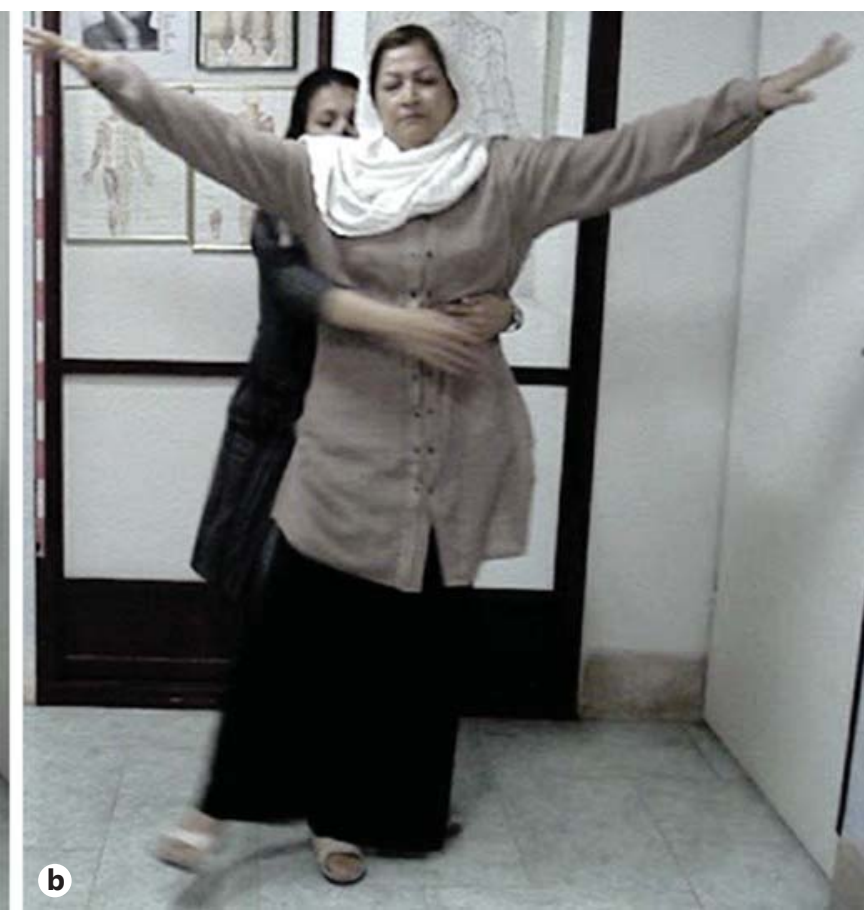

Fig. 4. Left deviation of patient (the patient is supported by her daughter). a Standard Romberg test. b Patient falling during the Romberg test.

Her right-side pulse was slippery and her left-side pulse was almost the same. She had a palpable pulse in every three positions (proximal, middle and distal) on both hands. Her pulse did not match with her syndrome differentiation, which could be explained by the fact that her pulse was showing her underlining disease (brucellosis), but her symptoms could be due to drug reaction. Concerning tongue diagnosis, the color was pink to red, with a normal shape. The coat was thin and white.

\section{Points}

Theacupuncture points wereasfollows:DU16,LI4,Liv3, DU20 and Ex-HN5(EACUP*3wire; fig. 1), SJ4, SJ5, ST34, ST36, ST37, ST40, GB20, SP6 and Ex-HN 3 (for 3 h) [5].

\section{Method}

We started acupuncture using needles $0.20 \mathrm{~mm}$ in diameter and $10 \mathrm{~mm}$ in length (Huanqiu, China). After puncturing the DU16 point and getting DE qi, we continued manipulation for $15 \mathrm{~s}$ and finally withdrew the needle [5]. On the other points, however, the needle remained for about 25-30 min. They were manipulated every $10 \mathrm{~min}$. The other points were manipulated with an even method. Then, the section of the Ex-HN 3 point was punctured, and the needle remained for $3 \mathrm{~h}$ (fig. 5) [5]. After getting a sensation of needling, the handles of needles (Ex-HN 5 and DU20) (fig. 1) were connected to a G6805-2 type electric stimulator with the frequency of $80 \mathrm{~Hz}$ (continuous wave and tolerable intensity).

\section{Treatment}

In one course, the needles were kept for 30 min once a day for 10 days. The time interval was 7-10 days between two courses. On Thursdays and Fridays, she had a rest, and the process lasted for 2 weeks of treatment (10 sessions). The standard course for this treatment 
Fig. 5. Place and puncture of ExHN 3.

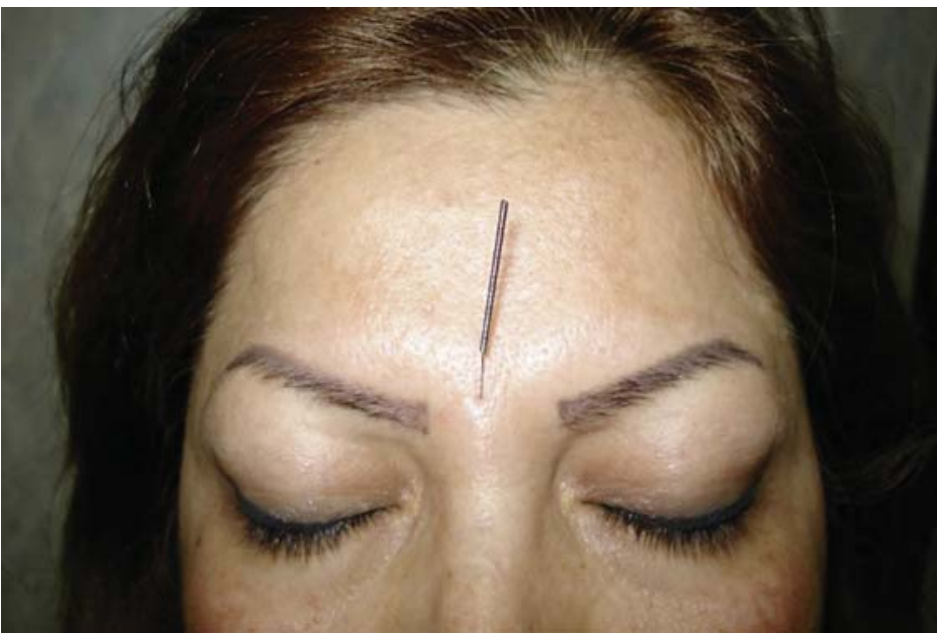

is between 15 and 20 sessions, but as the patient had a complete recovery, she did not wish to continue her treatment.

\section{Outcome}

The equilibrium examination after acupuncture was improved. The gait was normal; there was no action tremor or negative Romberg test. The patient's gait and even running were normal. When she was standing on both legs with her arms stretched out and eyes open, she could keep her body balanced without falling for $65 \mathrm{~s}$. In the same evaluation of equilibrium with closed eyes (negative Romberg test), she could stand for more than $65 \mathrm{~s}$. She achieved her body balance again and was symptom free after 10 days. This protocol of treatment is used for mind opening and gaining body balance [5]. The hair loss which the patient had had for several months also improved after the acupuncture treatment, which was an accidental finding. On the 2-year telephone follow-up, she did not report any problems and had no relapse of dizziness and/or gait disorder.

\section{Discussion}

\section{Brucellosis}

Human brucellosis is a well-known disease for its variety of clinical manifestations and complications. It is commonly characterized by clinical features that include fever, sweating, muscle pain, anorexia and arthralgia [8]. Involvement of the musculoskeletal system is the most common complication of brucellosis, and sometimes it is presented as FUO [1, 4, 9].

The finding of Brucella organisms on blood culture is diagnostic, but in many cases might be negative, as observed in our patient, probably due to intermittent bacteremia, where the bacillus is difficult to isolate [10]. The diagnosis may also be confirmed by demonstrating raised STA of Brucella in the blood [10]. The diagnostic criteria for brucellosis in an endemic region are a titer $\geq 1 / 320$ in STA and/or a titer $\geq 1 / 160$ in 2ME [11]. Several authors have mostly reported positive agglutination titers in cases of Brucella osteomyelitis; however, the diagnosis of brucellosis cannot be excluded even if the serum agglutination tests are negative $[4,12]$ (similar to this case report).

On the other hand, Brucella spondylitis is radiologically indistinguishable from spondylitis caused by other microorganisms [10]. In the list of differential diagnoses, tuberculosis 
spondylitis, osteomyelitis due to other hematogenous bacteria and even metastatic lesions should be considered [10]. Needle aspiration or exploration is frequently performed to aid in identification of the disease [10], which has been requested in this case to confirm brucellosis. The microorganism was not isolated from the L3 vertebral biopsy culture, but clinically the response to treatment with streptomycin, tetracycline and rifampin was highly suggestive of the presence of brucellosis.

In conclusion, in endemic areas brucellosis should be considered in the differential diagnosis of every FUO and destructive spinal lesion even if serology and microbiology are inconclusive.

\section{Acupuncture}

Vestibulopathy is a kind of vertigo, and TCM describes it as wind. The Chinese medical philosophy claims that 'no vertigo would happen without wind', 'no vertigo would happen without phlegm' and 'no vertigo would happen without deficiency'. Therefore, wind, phlegm and deficiency are the main causative reasons of vertigo, closely related to the liver, spleen and kidney [13]. In the presented patient, the deficiency of KI qi, SP yang and liver blood is the main underlying condition of wind attack. Deficiencies in the spleen and stomach for transforming and transporting water and food for production of qi and blood causes the blood and qi deficiency, and consequently there is no clear yang flowing to the brain. In the case of KI qi deficiency, the spleen yang is not nourished, and this can worsen the condition. Finally, the liver blood deficiency in our patient can be due to the underlying KI qi and spleen yang deficiency, which predisposed her to wind attack and vertigo $[14,15]$. The governor vessel is a 'sea of yang meridians'. Fengchi (GB20) is a crossing acupoint of the foot Shaoyang meridian and yang link vessel and is mainly used to treat vertigo and other symptoms like blurred vision and pain in the neck [13]. Ex-HN 3, Ex-HN 5, and DU16 are four points on the four sides of the head and are responsible for mind opening. Also DU20 and GB20 on the top and base of the head can enhance the qi rising and yang tonification in the brain and open the mind, respectively.

All yang channels of the body gather in the governor vessel, and its collaterals spread over the head and mind. Stimulation of DU20 and Ex-HN 5 (fig. 1) on the top and sides of the head (mind) can enhance the circulation of qi and blood on the meridians of the head, strengthen the brain and activate the collaterals [13]. In addition, stimulation of Ex-HN 3 for $3 \mathrm{~h}$ can regulate and stimulate the circulation of qi and blood in the brain [5].

The points on the front and back of the head and neck like Ex-HN 3, GV16 and GB20, and also points on the top and sides of the head like DU20 and Ex-HN 5 have the function of dredging the meridian, regulating yin-yang and qi-blood. It indicates the principle 'where the meridian passes, there the disease can be treated' [15]. In this case, the main meridians involved were Liv and GB (around the ear), and also the main meridian for brain (mind) nourishing, which is the DU meridian. The combination of these points can promote qi and blood circulation and raise the lucid yang to open the orifice, awaken the mind and expel wind to relieve the vertigo or vestibulopathy [16].

\section{Conclusion}

Today, with all the developments in Western medicine, there are still conditions that remain treatment resistant, for example vestibulopathy due to streptomycin, which cannot be treated. This report tries to show that some patients can be managed better with integrative medicine and acupuncture than with a monodimensional approach. However, this needs to be confirmed in further studies with a large sample size. 
Avijgan et al.: Integrative Medicine and Drug-Induced Vestibulopathy following Brucellosis Treatment: Clinical Practice

\section{Acknowledgements}

We gratefully acknowledge Mrs. Fatima Abdollahi for her final English editing of this article.

\section{Disclosure Statement}

There are no conflicts of interest related to this study.

\section{References}

1 Sakran W, Chazan B, Koren A: Brucellosis: clinical presentation, diagnosis, complications and therapeutic options. Harefuah 2006;145:836-840, 860.

2 Jokar MH, Pezeshkirad M: Brucella osteomyelitis of the pubic bones: report of two cases. Iran J Radiol 2009, 6:13-17.

3 Comprehensive Guideline of Communicable Diseases Surveillance System for Family Physician. Centers for Disease Control, Ministry of Health, 2013, pp 153-218.

4 Avijgan M, Hafizi M, Salemi A, Dehkordi SAI: Unusual presentation of brucellosis: afebrile, culture positive brucellosis and culture positive, seronegative brucellosis. Asian Pac J Trop Med 2009;2:22-27.

5 Zhao H, Liu Z, Zhao T, Zhao V, Wang L: Therapeutic effect observation on treatment of vestibular central balance disturbance with brain-regulating electro-acupuncture. J Acupunct Tuina Sci 2006;4:144-147.

6 WHO Western Pacific Region: WHO international standard terminologies on traditional medicine in the Western Pacific region. http://www.wpro.who.int/publications/docs/WHOIST_26JUNE_FINAL.pdf.

7 Avijgan M, Avijgan M: The infrastructure of the integrative human body; Qi/Dameh, Qi Movement/Rouh and Zheng/Mezadj - scientific base. Int J Integr Med 2013;1:2013.

8 Shehabi A, Shakir K, El-Khateeb M, Qubain H, Fararjeh N, Abu Shamat AR: Diagnosis and treatment of 106 cases of human brucellosis. J Infect 1990;20:5-10.

9 Ciftdoğan DY, Bayram N, Vardar F: Brucellosis as a cause of fever of unknown origin in children admitted to a tertiary hospital in the Aegean region of Turkey. Vector Borne Zoonotic Dis 2011;11:1037-1040.

10 Samra Y, Hertz M, Shaked S, Altman G: Brucellosis of the spine. A report of 3 cases. J Bone Joint Surg Br 1982; 64:429-431

11 Khorvash F, Keshteli AH, Behjati M, Salehi M, Emami Naeini A: An unusual presentation of brucellosis, involving multiple organ systems, with low agglutinating titers: a case report. J Med Case Rep 2007;1:53.

12 Yorgancigil H, Yayli G, Oyar O: Neglected case of osteoarticular Brucella infection of the knee. Croat Med J 2003; 44:761-763.

13 Zhang X: Treatment of 65 cases of cervical vertigo treated by acupuncture plus medication. J Acupunct Tuina Sci 2006;4:339-343.

14 Li P, Wu Y, Li H: Treatment of 30 cases of vertigo due to Qi-blood deficiency by pricking Jingbailao (Ex-HN 15) with fire needle therapy. J Acupunct Tuina Sci 2013;11:187-189.

15 Li H, Shen H, Li Y: Hemodynamic influence of arteria vertebralis type cervical spondylosis treated by needling Yuzhen (BL9) and Fengchi (GB20). J Acupunct Tuina Sci 2005;3:38-44.

16 Wu Z: Clinical applications of acupoints Baihui (GV 20) and Sishencong (Ex-HN 1). J Acupunct Tuina Sci 2010; 8:394-396. 